\title{
Azelnidipine attenuates glomerular damage in Dahl salt-sensitive rats by suppressing sympathetic nerve activity
}

\author{
Hajime Nagasu, Minoru Satoh, Sohachi Fujimoto, Naruya Tomita, Tamaki Sasaki and Naoki Kashihara
}

Dihydropyridine-type calcium channel blockers (CCBs) exert potent antihypertensive effects. The CCB azelnidipine decreases heart rate by suppressing sympathetic nerve activity, which affects afferent and efferent arterioles in the glomeruli. We examined whether azelnidipine can improve progressive glomerular injury in comparison with amlodipine by suppressing renal sympathetic nerve activity in Dahl salt-sensitive rats. Glomerular circulation in Dahl salt-sensitive rats was monitored with a charge-coupled device camera before and after administration of amlodipine $\left(0.5 \mathrm{mg} \mathrm{kg}^{-1}\right.$, bolus injection) or azelnidipine $\left(0.1 \mathrm{mg} \mathrm{kg}^{-1}\right.$, bolus injection). Systemic sympathetic nerve activity was also compared by analysis of heart rate variability with a telemetry blood pressure monitoring system after crossover administration of amlodipine $\left(1.0 \mathrm{mg} \mathrm{kg}^{-1}\right.$ per day) and azelnidipine $\left(3.0 \mathrm{mg} \mathrm{kg}^{-1}\right.$ per day) for 1 week. To investigate renoprotective effects, rats were treated with amlodipine $\left(1.0 \mathrm{mg} \mathrm{kg}^{-1}\right.$ per day) or azelnidipine ( $3.0 \mathrm{mg} \mathrm{kg}^{-1}$ per day) for 3 weeks with or without renal denervation. The efferent arteriole contracted in response to acute amlodipine but not azelnidipine treatment. The low frequency/high frequency ratio, an index of parasympathetic nerve activity, decreased in response to azelnidipine but not amlodipine treatment. In response to chronic treatment, proteinuria and glomerular injury improved to a greater extent with azelnidipine compared with amlodipine. The renoprotective effects of azelnidipine were diminished by renal denervation. Azelnidipine decreased glomerular damage in Dahl salt-sensitive rats to a greater extent than amlodipine. Azelnidipine appeared to decrease intraglomerular pressure by suppressing sympathetic nerve activity.

Hypertension Research (2012) 35, 348-355; doi:10.1038/hr.2011.184; published online 10 November 2011

Keywords: calcium channel blocker; chronic kidney disease; denervectomy; glomeruli

\section{INTRODUCTION}

Hypertension not only causes renal injury but is also a major progressive factor for organ damage in end-stage renal disease. ${ }^{1}$ Dihydropyridine-type calcium channel blockers (CCBs) exert potent antihypertensive effects and are commonly used in the treatment of hypertension. Their renoprotective effects have been demonstrated in many experimental models of hypertensive renal damage. ${ }^{2-4}$ Recent large-scale clinical studies have shown that long-acting, dihydropyridine-type CCBs effectively suppress the progression of renal damage. ${ }^{5,6}$ However, the predominant effect on preglomerular vessels might cause glomerular hypertension, which could ultimately be associated with the progression of kidney disease. Generally, CCBs dilate glomerular afferent arterioles more than efferent arterioles and can induce glomerular hypertension if the systemic blood pressure is not adequately low. ${ }^{7}$ Because glomerular hypertension can induce glomerular injury, the blood pressure must be decreased sufficiently to also decrease glomerular hypertension during CCB use. ${ }^{8}$
Azelnidipine is a long-acting, dihydropyridine-type CCB that decreases heart rate ${ }^{9}$ by suppressing sympathetic nerve activity. ${ }^{10}$ Its renoprotective effect has been demonstrated in several experimental models ${ }^{11}$ and in patients with hypertension. ${ }^{12}$ In hypertensive patients with mild chronic kidney disease, azelnidipine has a greater effect in decreasing proteinuria than does amlodipine. ${ }^{12}$ However, the mechanism underlying its renoprotective effect, including its effect on glomerular hemodynamics, has not been completely elucidated. Sympathetic nerve activity affects the afferent and efferent arterioles in the glomeruli and adjusts glomerular filtration; ${ }^{13,14}$ this may explain the renoprotective effects of azelnidipine in patients with hypertension.

We hypothesized that azelnidipine possesses renoprotective effects beyond its antihypertensive effect because it lowers intraglomerular pressure more so than other L-type CCBs. The aim of this study was to elucidate whether azelnidipine can improve progressive glomerular injury by suppressing renal sympathetic nerve activity and to compare 
the effects of azelnidipine with those of amlodipine. First, we compared the acute effects of azelnidipine and amlodipine on glomerular efferent and afferent arterioles in Dahl salt-sensitive rats. We then examined sympathetic nerve activity during crossover administration of azelnidipine and amlodipine. Finally, we investigated the effect of chronic administration of azelnidipine and amlodipine on renal function in rats with or without renal denervation.

\section{METHODS}

The protocols for this study (no. 08-004 and 08-068) were approved by the Animal Research Committee of Kawasaki Medical School and conducted according to the Guide for the Care and Use of Laboratory Animals of Kawasaki Medical School, which is based on the National Institutes of Health Guide for the Care and Use of Laboratory Animals (NIH publication no. 80-23, revised 1996).

\section{Acute administration of amlodipine and azelnidipine}

Male Dahl salt-sensitive rats ( 7 weeks old) were purchased from Clea Japan (Osaka, Japan). For the first 3 weeks, rats were fed an $8 \%$ salt diet to induce hypertension. For each rat, systolic blood pressure (SBP) was measured every week by the tail-cuff method with an automatic sphygmomanometer (BP98A; Softron, Tokyo, Japan). After confirming the induction of hypertension (SBP $>180 \mathrm{~mm} \mathrm{Hg}$ ), polyethylene catheters (PE50; Nihon Becton Dickinson, Tokyo, Japan) were inserted into the carotid artery under sevoflurane-induced anesthesia to monitor SBP and heart rate by a pressure transducer (RMP-6000; Nihon Kohden, Tokyo, Japan). Next, a catheter was inserted into the left external jugular vein for infusion of amlodipine $\left(0.5 \mathrm{mg} \mathrm{kg}^{-1}\right.$, bolus injection; $n=5$, Sigma-Aldrich Japan, Tokyo, Japan) or azelnidipine $\left(0.1 \mathrm{mg} \mathrm{kg}^{-1}\right.$, bolus injection; $n=5$, provided by Daiichi Sankyo, Tokyo, Japan). The dosage of amlodipine and azelnidipine was decided in reference to the procedure of Shokoji et al. ${ }^{15} \mathrm{~A}$ dorsal incision was then made under sterile conditions, and the left kidney was exteriorized. Glomerular microcirculation was recorded for 30 min after infusion by a charge-coupled device videomicroscope placed in direct contact with the exposed surface of the kidney, as described previously. ${ }^{11}$ Sequential images of the renal microvessels were digitized with a freeze-frame modality. Diameters of afferent and efferent arterioles were measured with Image-J software (http://rsbweb.nih.gov/ij/).

\section{Crossover administration of amlodipine and azelnidipine}

Male Dahl salt-sensitive rats ( 7 weeks old) were used. For heart rate variability analysis, a telemetry blood pressure monitoring system was used. Under sevoflurane-induced anesthesia, a telemetry device (model TA11-PAC40; Data Sciences International, St Paul, MN, USA) was implanted into the abdominal aortas of eight rats. In brief, a midline abdominal incision was made, and the abdominal aorta was exposed with the use of sterile cotton swabs. The catheter of the telemetry device was inserted into the abdominal aorta and guided upstream. Animals were allowed 1 week for recovery before use in experiments. The rats were housed in a temperature- and humidity-controlled room with a 14:10-h light:dark cycle (lights on from 0700 to 2100 hours). For the first 3 weeks, the rats were fed an $8 \%$ salt diet to induce hypertension. After the induction of hypertension was confirmed (SBP $>180 \mathrm{~mm} \mathrm{Hg}$ ), rats were treated with amlodipine $\left(1.0 \mathrm{mg} \mathrm{kg}^{-1}\right.$ per day; $\left.n=4\right)$ or azelnidipine $\left(3.0 \mathrm{mg} \mathrm{kg}^{-1}\right.$ per day; $n=4$ ) by gavage for 1 week. The dosage of amlodipine and azelnidipine were decided by performing preliminary experiment in reference to a clinical dose. ${ }^{12,16}$ They were then left untreated for a 1 week wash-out period. The rats were then treated with the other drug for 1 week. Mean blood pressure and heart rate were recorded by the telemetry system (Dataquest ART 2.1 Gold Telemetry System; Data Sciences International; with DSI PhysioTel RPC-1 receivers connected to a DSI Data Exchange Matrix) every 5 min (each recording representing the average of pressure measurements made during 60 heartbeat periods over 10-s interval) continuously for $24 \mathrm{~h}$ on the last day of drug administration. This crossover experimental protocol was illustrated in Figure 1. Data from each animal were exported (Dataquest ART analysis software; Data Sciences International) to a spreadsheet program (Excel 2003; Microsoft Corporation, Seattle, WA, USA). Systemic sympathetic nerve activity

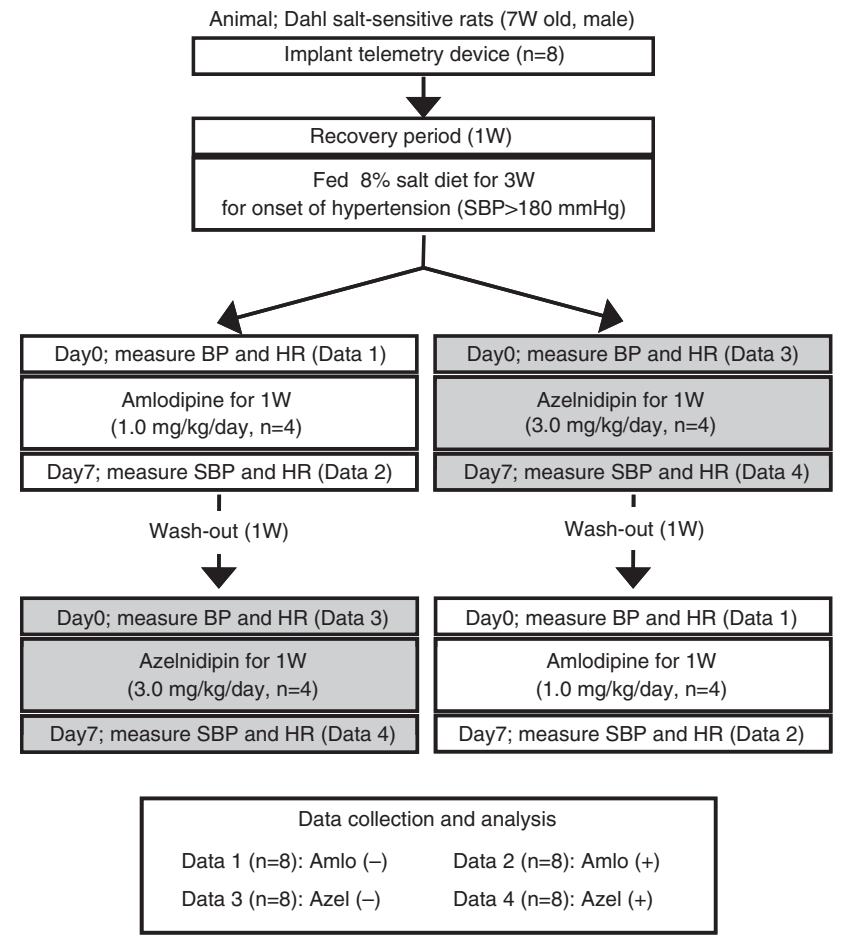

Figure 1 Diagram of crossover administration experiment. Amlo (-), before treatment with Amlo; Amlo (+), after treatment with Amlo; Azel ( - ), before treatment with Azel; Azel (+), after treatment with Azel. BP, blood pressure; $\mathrm{HR}$, heart rate.

was examined by heart rate variability analysis with the telemetry blood pressure monitoring system and using the low frequency (LF) to high frequency (HF) ratio as an index of parasympathetic nerve activity. ${ }^{17}$ As reported previously, ${ }^{18,19}$ spectral analysis of short-term recordings is characterized by two major components at low $(0.05-0.15 \mathrm{~Hz})$ and high $(0.15-0.40 \mathrm{~Hz})$ frequency. The LF component has been proposed ${ }^{17}$ and used, particularly when expressed in normalized units, as an index of sympathetic modulation, whereas HF, which is a measure of respiratory sinus arrhythmia, is used as an index of vagal activity. The ratio of the absolute powers of the LF and HF components was also calculated, and the LF/HF ratio was used as an index of sympathovagal interaction. ${ }^{17}$

\section{Chronic administration of amlodipine and azelnidipine}

Male Dahl salt-sensitive rats ( 7 weeks old) fed an $8.0 \%$ salt diet were assigned to one of three groups: the DH group $(n=7)$ composed of untreated hypertensive rats; the DH-Amlo group $(n=7)$ composed of hypertensive rats treated with amlodipine $\left(1.0 \mathrm{mg} \mathrm{kg}^{-1}\right.$ per day) and the DH-Azel group $(n=7)$ composed of hypertensive rats treated with azelnidipine $\left(3.0 \mathrm{mg} \mathrm{kg}^{-1}\right.$ per day). We also included a group of normotensive Dahl salt-sensitive rats fed a $0.9 \%$ salt diet (Dahl salt-sensitive rats fed a normal-salt diet (DN);n=7). To examine the effects of sympathetic nerve activity on renal injury, some DH-Amlo and DHAzel group rats ( $n=7$ in each group) had undergone bilateral renal denervation 1 week earlier (at 6 weeks of age). Renal denervation was performed as described previously. ${ }^{20}$ The SBP and heart rate were measured every week by the tail-cuff method. After 3 weeks, urine was collected over $24 \mathrm{~h}$ to measure proteinuria and oxidative stress marker, 8-hydroxydeoxyguanosine (8-OHdG) and malondialdehyde (MDA), and each rat was anesthetized with sevoflurane. 8-OHdG and MDA were measured by the enzyme-linked immunosorbent assay kit (New 8-OHdG Check ELISA, Japan Institute for the Control of Aging, Shizuoka, Japan, and the Bioxytech MDA Assay Kit, Percipio Biosciences, Portland, OR, USA, respectively). Blood was drawn via cardiac puncture to measure serum creatinine. Half of each kidney was cut in cross-section and fixed in $4 \%$ paraformaldehyde, and the remaining half was used for the preparation of RNA and protein assay. Coronal sections of the kidney $(4-\mu \mathrm{m}$ 
thick) were stained with periodic acid-Schiff and examined in a blind fashion by light microscopy to assess glomerular and arterial morphological characteristics. Glomerular injury score was calculated as described previously. ${ }^{21}$ At least 30 glomeruli were selected randomly from each rat. The medial thickness-tolumen ratio was calculated as described previously. ${ }^{22}$ Five regions of the interlobular artery from each rat were evaluated, and the average ratio was calculated. Proteinuria and creatinine levels in serum and urine were measured by SRL (Tokyo, Japan). A piece of kidney tissue was used for measurement of norepinephrine concentration as described previously. ${ }^{20}$ Total RNA isolation, reverse transcriptase reactions and real-time quantitative polymerase chain reactions for angiotensinogen and angiotensin type 1 receptor were performed as described previously. ${ }^{23}$ The change in the expression was expressed by standardizing RNA levels corrected for glyceraldehyde-3-phosphate dehydrogenase expression in the sample.

\section{Statistical analysis}

Values are presented as mean \pm s.e.m. All parameters except those for crossover administration of amlodipine and azelnidipine were evaluated by two-tailed unpaired Student's $t$-test, Welch $t$-test or Mann-Whitney $U$-test, or by 1 -factor analysis of variance with the Tukey-Kramer test for multiple comparisons. Mean blood pressure, heart rate and LF/HF ratio data from the crossover administration of amlodipine and azelnidipine were evaluated by 1 -factor repeated measures analysis of variance. A $P$-value $<0.05$ denoted statistical significance.

\section{RESULTS}

Effects of acute administration of amlodipine and azelnidipine on glomerular microcirculation

At 20 min after injection with amlodipine or azelnidipine, SBP decreased by the same amount for both drugs (Figure 2a; amlodipine, $184 \pm 7-142 \pm 5 \mathrm{~mm} \mathrm{Hg}$; azelnidipine, $180 \pm 6-140 \pm 5 \mathrm{~mm} \mathrm{Hg}$ ). Heart rate was unchanged in amlodipine-treated rats (Figure $2 b ; 375 \pm 3$ $384 \pm 3$ b.p.m.), but that in azelnidipine-treated rats decreased significantly in comparison (to $330 \pm 8$ b.p.m.; $P<0.05$ vs. amlodipine treatment). To compare the acute effects of amlodipine and azelnidipine on glomerular microcirculation, glomerular afferent and efferent arterioles were monitored with a charge-coupled device camera (Figure 2c). The diameter of the afferent arterioles was unchanged in both groups at $20 \mathrm{~min}$ after injection (Figure 2d). However, the efferent arteriole was significantly more contracted in response to amlodipine compared with azelnidipine (Figure 2d), indicating that the intraglomerular pressure may be decreased to a greater extent in the azelnidipine group than in the amlodipine group at the same blood pressure.

\section{Effects of amlodipine and azelnidipine on systemic sympathetic} nerve activity

In the crossover administration experiment, systemic sympathetic nerve activity was examined by heart rate variability analysis conducted with a telemetry blood pressure monitoring system. Mean blood pressure was decreased the same amount in the amlodipine

Figure 2 Changes in heart rate after and glomerular microcirculation acute injection of amlodipine or azelnidipine. Systolic blood pressure (a) and heart rate (b) were recorded for $30 \mathrm{~min}$ after amlodipine (Amlo; $0.5 \mathrm{mg} \mathrm{kg}^{-1}$ ) or azelnidipine (Azel; $0.1 \mathrm{mg} \mathrm{kg}^{-1}$ ) injection (i.v. in Dahl salt-sensitive rats under anesthesia. $n=5$ for each group. ${ }^{*} P<0.05$ vs. Amlo. (c) Morphological changes in glomeruli before and 20 min after injection of amlodipine or azelnidipine in Dahl salt-sensitive rats under anesthesia. Af, afferent arteriole; Ef, efferent arteriole. (d) Changes in afferent and efferent arteriole diameter after injection of amlodipine (Amlo) or azelnidipine (Azel). $n=5$ for each group. ${ }^{*} P<0.05$ vs. before treatment; ${ }^{\dagger} P<0.05$ vs. Amlo.
$(148 \pm 3-130 \pm 3 \mathrm{~mm} \mathrm{Hg})$ and azelnidipine $(148 \pm 3-129 \pm 4 \mathrm{~mm} \mathrm{Hg})$ groups (Figures $3 \mathrm{a}-\mathrm{c}$ ). In contrast, heart rate (Figures $3 \mathrm{~d}-\mathrm{f}$ ) was unchanged in response to amlodipine treatment (355 $\pm 12-$ $350 \pm 9$ b.p.m.) but was significantly decreased in response to azelnidipine treatment (357 $\pm 11-319 \pm 9$ b.p.m.). In addition, the LF/HF ratio was decreased in response to azelnidipine but not to amlodipine

a Systolic blood pressure

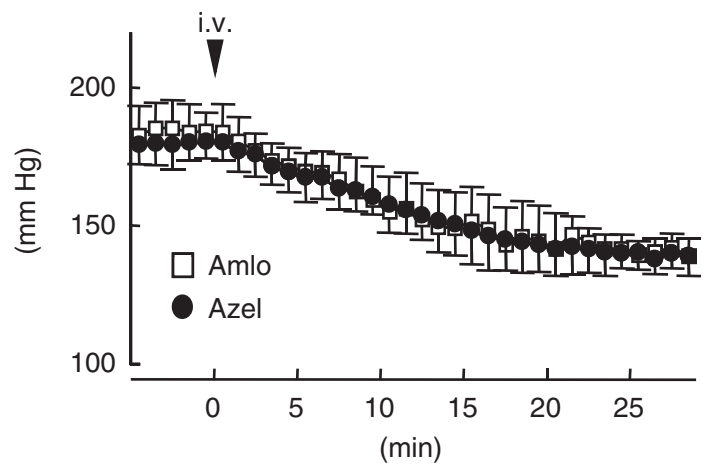

b

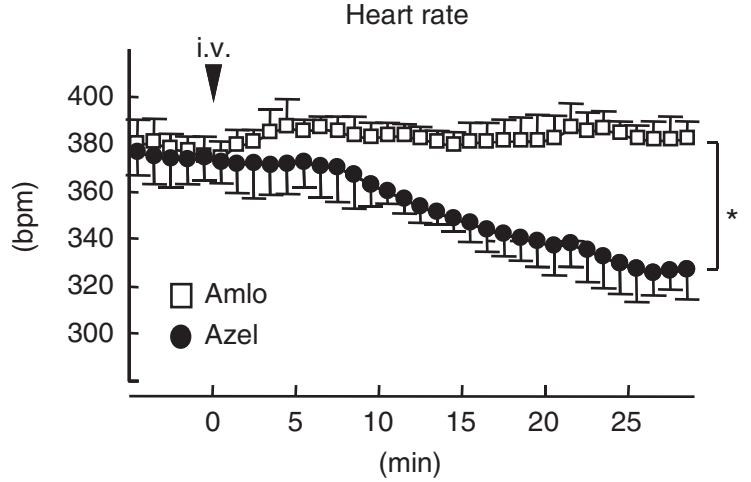

C

을 Pretreatment after $20 \mathrm{~min}$

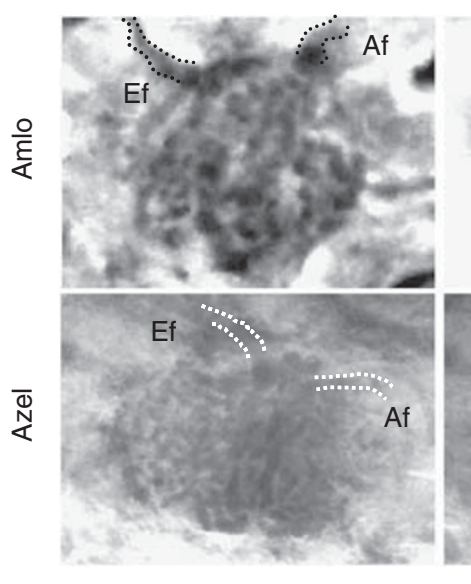

d

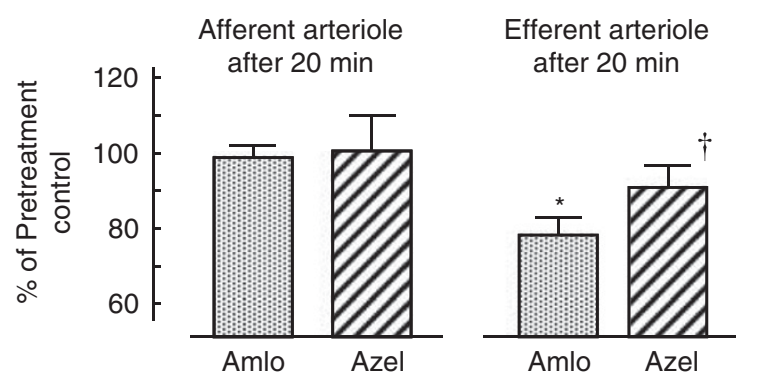



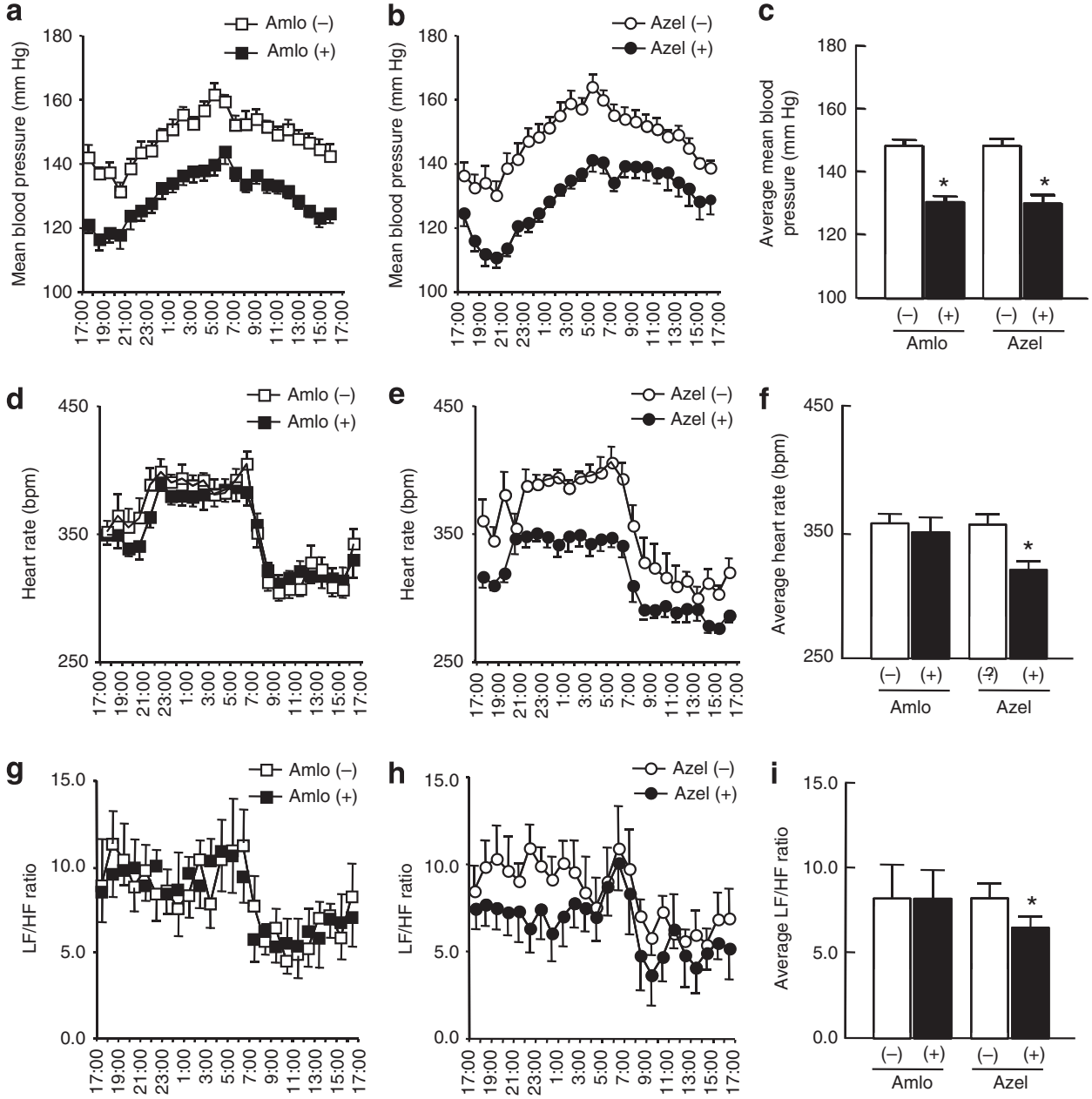

Figure 3 Mean blood pressure, heart rate and sympathetic nerve activity after crossover administration of amlodipine or azelnidipine. Daily changes in mean blood pressure, heart rate, and LF/HF ratio before and after 1 week of treatment with (a, d, g) amlodipine (Amlo; $1.0 \mathrm{mg} \mathrm{kg}^{-1}$ per day) or (b, e, h) azelnidipine (Azel; $3.0 \mathrm{mg} \mathrm{kg}^{-1}$ per day) in Dahl salt-sensitive rats. Amlo (-), before treatment with Amlo; Amlo (+), after treatment with Amlo; Azel (-), before treatment with Azel; Azel (+), after treatment with Azel. (c) Average mean blood pressure. (f) Average heart rate. (i) Average LF/HF ratio. $n=8$ for each group. ${ }^{*} P<0.05$ vs. untreatment.

Table 1 Pathophysiological data for Dahl salt-sensitive rats treated with amlodipine or azelnidipine

\begin{tabular}{|c|c|c|c|c|}
\hline Group & $D N(\mathrm{n}=7)$ & $D H(\mathrm{n}=7)$ & $D H-A m / O(n=7)$ & DH-Azel $(\mathrm{n}=7)$ \\
\hline $\mathrm{SBP}(\mathrm{mm} \mathrm{Hg})$ & $124 \pm 4$ & $201 \pm 5^{a}$ & $156 \pm 7^{a, b}$ & $156 \pm 7^{a, b}$ \\
\hline Kidney NE (ng g ${ }^{-1}$ tissue) & $0.65 \pm 0.06$ & $1.55 \pm 0.10^{\mathrm{a}}$ & $1.03 \pm 0.06^{a, b}$ & $0.76 \pm 0.06^{b, c}$ \\
\hline Kidney AGT mRNA & $1.00 \pm 0.05$ & $2.11 \pm 0.19^{a}$ & $1.43 \pm 0.09^{a, b}$ & $1.39 \pm 0.13^{a, b}$ \\
\hline Kidney AT1R mRNA & $1.00 \pm 0.04$ & $0.71 \pm 0.10^{\mathrm{a}}$ & $0.86 \pm 0.08^{a}$ & $0.81 \pm 0.05^{a}$ \\
\hline
\end{tabular}

Data are presented as mean \pm s.e.m.

AGT, angiotensinogen; AT1R, angiotensin type 1 receptor; BW, body weight; DN, untreated Dahl salt-sensitive hypertensive rats fed a $0.9 \%$ salt diet; DH, untreated Dahl salt-sensitive hypertensive rats fed an $8 \%$ salt diet; DH-Amlo, Dahl salt-sensitive hypertensive rats treated with amlodipine $\left(1.0 \mathrm{mg} \mathrm{kg}^{-1} \mathrm{per}_{\text {day); }} \mathrm{DH}\right.$-Azel, Dahl salt-sensitive hypertensive rats treated with azelnidipine (3.0 $\mathrm{mg} \mathrm{kg}^{-1}$ per day); HR, heart rate; NE, norepinephrine concentration; SBP, systolic blood pressure.

${ }^{\mathrm{a}} \mathrm{P}<0.05$ vs. DN; ${ }^{\mathrm{b}} \mathrm{P}<0.05$ vs. $\mathrm{DH} ;{ }^{\mathrm{C}} \mathrm{P}<0.05$ vs. DH-Amlo.

(Figures $3 \mathrm{~g}-\mathrm{i}$ ). These data indicated that systemic sympathetic nerve activity is suppressed by azelnidipine treatment.

\section{Effects of chronic administration of amlodipine and azelnidipine} on renal and vascular damage

For the chronic administration experiment, rats were treated with amlodipine $\left(1.0 \mathrm{mg} \mathrm{kg}^{-1}\right.$ per day) or azelnidipine $\left(3.0 \mathrm{mg} \mathrm{kg}^{-1}\right.$ per day) for 3 weeks. The SBP decreased significantly in both groups compared with the nontreated DH group (Dahl salt-sensitive rats fed a high-salt diet), and it was also higher compared with the DN group (Table 1). Heart rate decreased in the azelnidipine-treated DH group and was similar to that in the DN group (Table 1). Kidney norepinephrine concentration was significantly increased in the DH group compared with the DN group and was much more decreased in the 

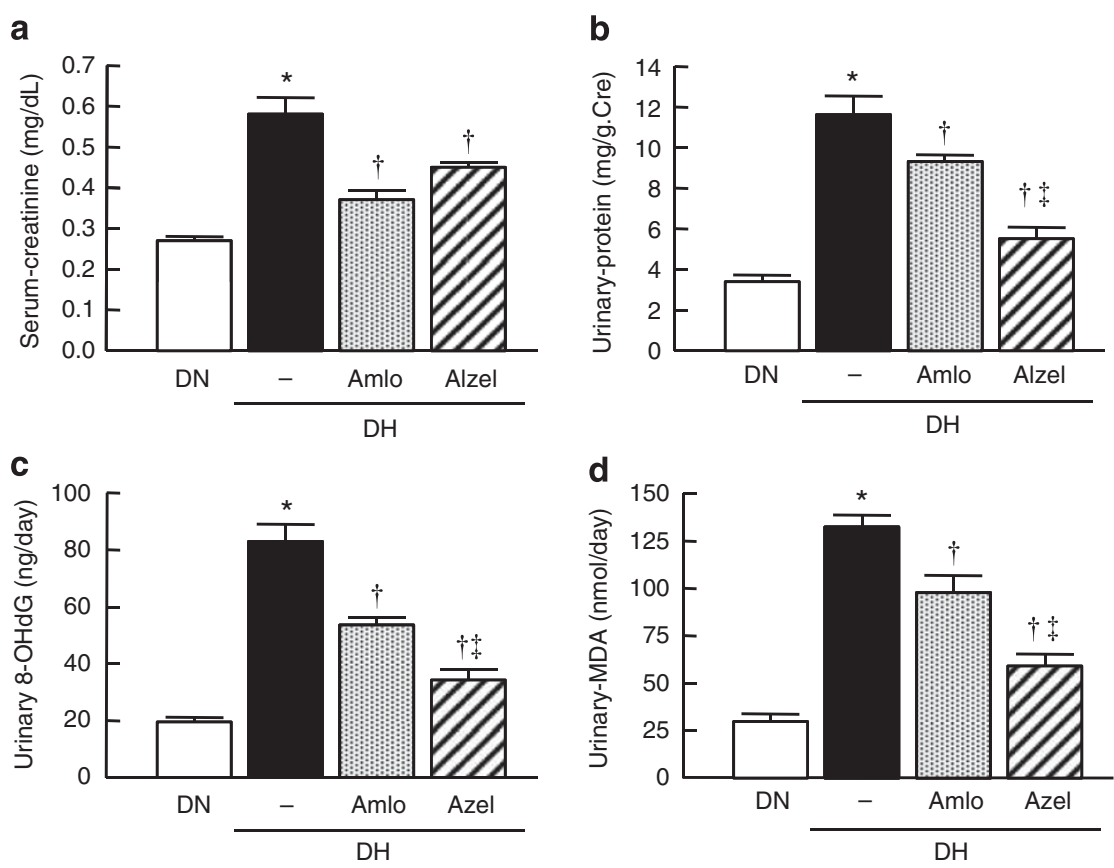

Figure 4 Changes in renal function after chronic administration of amlodipine (Amlo) or azelnidipine (Azel) in Dahl salt-sensitive rats. Serum creatinine (a), urinary protein (b), urinary $8-\mathrm{OHdG}$ (c) and urinary MDA (d). $n=7$ for each group. ${ }^{*} P<0.05$ vs. DN; ${ }^{\dagger} P<0.05$ vs. $\mathrm{DH} ;{ }^{\ddagger} P<0.05$ vs. DH-Amlo. DN, control normotensive Dahl salt-sensitive rats; DH, hypertensive Dahl salt-sensitive rats; MDA, malondialdehyde; 8-OHdG, 8-hydroxydeoxyguanosine.

azelnidipine group compared with the amlodipine group (Table 1), indicating that azelnidipine had a greater effect on sympathetic nerve activity than amlodipine, irrespective of its blood pressure-lowering effect.

Increased angiotensinogen mRNA in DH group decreased in both the azelnidipine-treated $\mathrm{DH}$ group and the amlodipine-treated $\mathrm{DH}$ group at same degree (Table 1). Similarly, decreased angiotensin type 1 receptor mRNA in DH group increased in both the azelnidipinetreated $\mathrm{DH}$ group and the amlodipine-treated $\mathrm{DH}$ group at same degree (Table 1), indicating that there was no difference in azelnidipine and amlodipine in regard with renal rennin-angiotensin system activity. The serum creatinine level was increased in the DH group compared with the DN group, and both amlodipine and azelnidipine decreased the serum creatinine level (Figure 4a). The serum creatinine level was slightly higher, but not significantly so, in the azelnidipine group compared with the amlodipine group. Urinary protein excretion decreased in both groups, but the azelnidipine group showed a lower urinary protein level than the amlodipine group (Figure $4 \mathrm{~b}$ ). These data indicated that azelnidipine decreased intraglomerular pressure more so than amlodipine, independent of its blood pressure-lowering effect. In addition, oxidative stress marker, 8-OHdG and MDA urinary excretion were also decreased in both azelnidipine and amlodipine-treated groups, but the azelnidipine group showed lower urinary levels than the amlodipine group. These data indicated that anti-oxidant effects by azelnidipine are superior compared with amlodipine. Less glomerular morphological change was noted in the azelnidipine group compared with the amlodipine group (Figure 5a). Glomerular mesangial expansion was not completely inhibited by amlodipine treatment. Glomeruli in the azelnidipine group did not show progressive injury. The glomerular injury score was higher in the $\mathrm{DH}$ group than in the $\mathrm{DN}$ group $(2.5 \pm 0.1$ vs. $0.7 \pm 0.2$, respectively; $P<0.05)$. Although amlodipine treatment significantly decreased the injury score (to $1.5 \pm 0.2 ; P<0.05 v s$. $\mathrm{DH}$ ), azelnidipine decreased it to a much greater extent (to $1.0 \pm 0.1 ; P<0.05 v s$. DH-Amlo). These data indicated that the glomerular protective effect of azelnidipine was independent of blood pressure, at least in part. To assess changes in small arteries, a parameter of hypertension-related vascular damage, the wall-to-lumen ratio was examined (Figure 5b). The medial thickness-to-lumen diameter ratio was higher in the DH group than in the DN group (DN, $0.19 \pm 0.03 ; \mathrm{DH}, 0.51 \pm 0.05 ; P<0.05$ ). Treatment with amlodipine or azelnidipine significantly decreased the ratio to the same degree (DH-Amlo, $0.30 \pm 0.02$; DH-Azel, $0.32 \pm 0.02$; $P<0.05$ vs. DH). These data indicated that vascular injury in Dahl salt-sensitive hypertensive rats depended on blood pressure.

Effect of renal denervation in rats receiving chronic administration of amlodipine and azelnidipine

To examine the effect of sympathetic nerve activity, the same experimental protocol was performed with Dahl salt-sensitive rats that had undergone renal denervation. Renal denervation was confirmed by assessment of renal tissue norepinephrine concentration (Table 2). No significant differences in proteinuria or glomerular damage were found between the azelnidipine group and the amlodipine group in rats subjected to renal denervation (Table 2), indicating that the renoprotective effect of azelnidipine depended on suppression of sympathetic nerve activity.

\section{DISCUSSION}

The aim of this study was to determine whether azelnidipine can improve progressive glomerular injury by suppressing renal sympathetic nerve activity. Our hypothesis was that azelnidipine exhibits renoprotective effects beyond its antihypertensive effect by lowering intraglomerular pressure more so than other L-type CCBs. Our results showed that azelnidipine suppressed sympathetic nerve activity when administered chronically, chronic treatment with azelnidipine attenuated renal dysfunction to a greater extent than chronic treatment with 


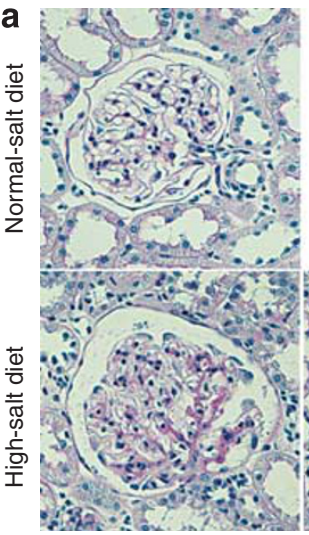

No treatment

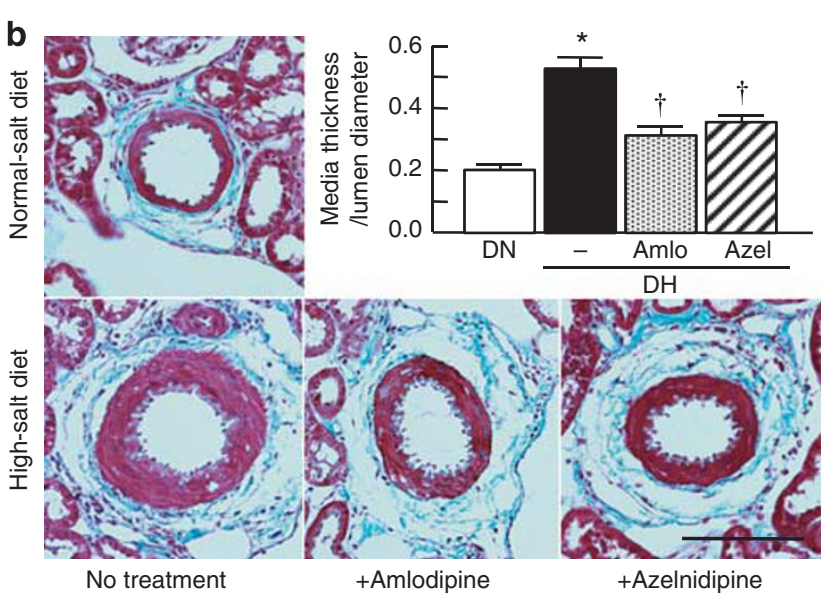

Figure 5 Changes in the glomeruli and interlobular artery after chronic administration of amlodipine (Amlo) or azelnidipine (Azel) in Dahl saltsensitive rats. (a) Periodic acid-Schiff staining. Scale bar $=100 \mu \mathrm{m}$. Glomerular injury caused by mesangial expansion was graded from 0 to 4 . More than 50 glomeruli were scored from each rat. After the scores from each rat were averaged, group means were calculated and compared. ${ }^{*} P<0.05$ vs. DN; $\uparrow^{P}<0.05$ vs. $\mathrm{DH} ; \ddagger P<0.05$ vs. DH-Amlo. DN, control normotensive Dahl salt-sensitive rats; $\mathrm{DH}$, untreated hypertensive Dahl saltsensitive rats. (b) Masson staining. Scale bar $=100 \mu \mathrm{m}$. The interlobular artery was identified as a single muscular artery within the inner cortex and, at times, lying close to the glomerulus. Five arteries were examined from each rat. After the medial thickness-to-lumen ratio was averaged for each rat, group means were calculated and compared.

Table 2 Pathophysiological data for renal denervated Dahl salt-sensitive rats treated with amlodipine or azelnidipine

\begin{tabular}{|c|c|c|}
\hline Group & $\begin{array}{l}\text { Amlo+renal denervation } \\
\qquad(\mathrm{n}=7)\end{array}$ & $\begin{array}{c}\text { Azel+renal denervation } \\
\qquad(\mathrm{n}=7)\end{array}$ \\
\hline BW (g) & $328 \pm 7$ & $324 \pm 8$ \\
\hline $\mathrm{SBP}(\mathrm{mm} \mathrm{Hg})$ & $159 \pm 6$ & $153 \pm 7$ \\
\hline HR (b.p.m.) & $404 \pm 9$ & $379 \pm 10$ \\
\hline $\begin{array}{l}\text { Kidney NE (ng } \text { ( }^{-1} \\
\text { tissue) }\end{array}$ & $0.16 \pm 0.09$ & $0.19 \pm 0.08$ \\
\hline S-Cre (mg dl-1) & $0.44 \pm 0.07$ & $0.52 \pm 0.02$ \\
\hline U-Pro (mg g-1 U-Cre) & $4.4 \pm 0.5$ & $5.1 \pm 0.9$ \\
\hline Glomerular injury score & $1.2 \pm 0.5$ & $1.1 \pm 0.5$ \\
\hline
\end{tabular}

Data are presented as mean \pm s.e.m.

Amlo, amlodipine; Azel, azelnidipine; BW, body weight; HR, heart rate; NE, norepinephrine concentration; SBP, systolic blood pressure. S-Cre, serum creatinine; U-Cre, urinary creatinine; U-Pro, urinary protein. amlodipine and that these effects were attenuated by renal denervation. These results suggest that the sympathoinhibitory effects of azelnidipine produced renoprotective effects in hypertensive rats.

To identify the effects of azelnidipine on the glomerular microcirculation, we used an intravital needle-type charge-coupled device camera videomicroscope. ${ }^{11}$ Using this type of system, Honda et al. ${ }^{24}$ compared the effects of nifedipine (L-type CCB) and efonidipine (L/T-type CCB) on the renal microvasculature. They showed that efonidipine potently relaxed both afferent and efferent arterioles with nearly the same activity. Both efonidipine and cilnidipine have been shown to protect glomeruli and decrease proteinuria in patients with untreated essential hypertension and chronic glomerulonephritis. ${ }^{25}$ We found that amlodipine, but not azelnidipine, resulted in efferent arteriole contraction in Dahl salt-sensitive rats, and SBP decreased to the same degree with both drugs. This finding indicated that azelnidipine may decrease intraglomerular pressure to a greater extent than amlodipine, which is beneficial for glomerular protection. Generally, L-type CCBs dilate afferent arterioles in glomeruli because L-type calcium channels are abundant in afferent arterioles. However, amlodipine did not dilate afferent arterioles but caused contraction of the efferent arteriole in this study. We used Dahl salt-sensitive rats as a model for hypertensive renal injury. These rats have been reported to show diminished myogenic responsiveness of afferent arterioles. ${ }^{26}$ Therefore, these rats may not be sensitive to CCBs in this context. In addition, renal sympathetic nerve activity has been shown to be increased in Dahl salt-sensitive rats. ${ }^{27}$ So efferent arterioles may undergo vasoconstriction by renal sympathetic nerve activation in response to a fall in systemic blood pressure by amlodipine treatment.

We also showed that azelnidipine, but not amlodipine, decreased sympathetic nerve activity in Dahl salt-sensitive rats, as determined by heart rate variability analysis and assessment of renal norepinephrine concentration. In general, dihydropyridine-type CCBs have been shown to cause reflex sympathetic stimulation and tachycardia associated with decreased blood pressure. ${ }^{28}$ The effect of azelnidipine on the suppression of sympathetic nerve activity appears to be a result of its strong antioxidant effect. The potent ability to decrease oxidative stress is a special property of azelnidipine..$^{29,30}$ We demonstrated that azelnidipine decrease urinary excretion of $8-\mathrm{OHdG}$ and MDA, oxidative stress marker, in Dahl salt-sensitive rats. Excessive oxidative stress in the brain regions stimulates sympathetic nerve activity and increases arterial pressure. ${ }^{31,32}$ Azelnidipine has been shown to decrease sympathetic nerve activity in the rostral ventrolateral medulla via its antioxidant effect. ${ }^{10}$ Hirooka et al. ${ }^{33}$ reported that amlodipine also decreases the generation of reactive oxygen species in several areas of the brain and decreases sympathetic nerve activity in stroke-prone, spontaneously hypertensive rats. However, another report suggested that amlodipine treatment increases sympathetic nerve activity. ${ }^{34}$ In this study, we could not confirm a sympathetic nerve activitydecreasing effect of amlodipine in Dahl salt-sensitive rats.

The use of $\beta$-blockers can reduce heart rate similarly to azelnidipine. However, lowering blood pressure with nonselective or selective $\beta$-blockers is associated with compensatory stimulation of the sympathetic nervous system and renin-angiotensin systems, leading to elevated norepinephrine and renin levels. ${ }^{35}$ Subsequent activation of vascular $\alpha$-adrenergic receptors results in an increase in systemic as well as renal vascular resistance. ${ }^{35}$ The sympathetic nervous system, the presumed main target of $\beta$-blocker activity, is one of the central pathways in the pathophysiology of hypertension. However, the nonselective $\beta$-blocker diminishes renal perfusion by lowering cardiac output and renal perfusion pressure, so it may adversely affect the renal function. On the other hands, though azelnidipine reduces blood 
pressure, it increases the renal blood flow. ${ }^{11}$ Azelnidipine may be a promising 'renal protective CCB' for the prevention of renal damages by suppressing sympathetic nerve activity in patients with hypertension.

As a matter of course, standard treatment for patients with chronic kidney disease includes an angiotensin-converting enzyme inhibitor or angiotensin II receptor blocker because these drugs may help prevent the progression of kidney failure. The target blood pressure for patients with chronic kidney disease is $<130 / 80 \mathrm{~mm} \mathrm{Hg}$. This target cannot be achieved in many patients by treatment with a single drug, and multidrug therapy is often required. ${ }^{36}$ Most clinical trials involving patients with hypertension have shown that treatment with multiple antihypertensive medications is necessary to attain the recommended blood pressure goals. ${ }^{37}$ In the ACCOMPLISH study, concomitant use of rennin-angiotensin system inhibitors and CCBs decreased cardiovascular events more effectively than concomitant use of rennin-angiotensin system inhibitors and diuretics. ${ }^{38}$ Among the dihydropyridine-type CCBs, azelnidipine has been reported to be superior to amlodipine in decreasing proteinuria in hypertensive patients with mild chronic kidney disease. ${ }^{12}$ Our present data indicate that the renoprotective effect of azelnidipine depends on its ability to suppress sympathetic nerve activity. The exact renoprotective effect of CCBs remains controversial. Nevertheless, the use of antihypertensive agents such as azelnidipine, cilnidipine ${ }^{39}$ and efonidipine ${ }^{40}$ to inhibit sympathetic nerve activity may be recommended because patients with chronic kidney disease exhibit sympathetic hyperactivity, ${ }^{41}$ which contributes to the pathogenesis of renal hypertension.

In conclusion, our present results showed that azelnidipine was superior to amlodipine in decreasing proteinuria and improving glomerular damage in Dahl salt-sensitive rats, irrespective of the blood pressure-lowering effect of the drug. These effects of azelnidipine may result from a decrease in intraglomerular pressure via suppression of sympathetic nerve activity. These findings suggest that azelnidipine may be a useful antihypertensive agent for patients with hypertensive kidney disease.

\section{CONFLICT OF INTEREST}

The authors declare no conflict of interest.

\section{ACKNOWLEDGEMENTS}

This study was supported by a Grant-in-Aid for Scientific Research from the Japan Society for the Promotion of Science (no. 21591047 to NK). We thank Ms Satomi Hanada, Ms Etsuko Yorimasa, Ms Keiko Ehara, Ms Asuka Maeda and Ms Miki Ishihara (Kawasaki Medical School) for their help with animal care and technical assistance. Azelnidipine was provided by Daiichi Sankyo (Tokyo, Japan).

1 Bakris GL, Williams M, Dworkin L, Elliott WJ, Epstein M, Toto R, Tuttle K, Douglas J, Hsueh W, Sowers J. Preserving renal function in adults with hypertension and diabetes: a consensus approach. National Kidney Foundation Hypertension and Diabetes Executive Committees Working Group. Am J Kidney Dis 2000; 36: 646-661.

2 Dworkin LD, Benstein JA, Parker M, Tolbert E, Feiner HD. Calcium antagonists and converting enzyme inhibitors reduce renal injury by different mechanisms. Kidney Int 1993; 43: 808-814.

3 Wenzel UO, Troschau G, Schoeppe W, Helmchen U, Schwietzer G. Adverse effect of the calcium channel blocker nitrendipine on nephrosclerosis in rats with renovascular hypertension. Hypertension 1992; 20: 233-241.

4 Ma G, Allen TJ, Cooper ME, Cao Z. Calcium channel blockers, either amlodipine or mibefradil, ameliorate renal injury in experimental diabetes. Kidney Int 2004; 66: 1090-1098.

5 Salvetti A, Ghiadoni L. Guidelines for antihypertensive treatment: an update after the ALLHAT study. J Am Soc Nephrol 2004; 15(Suppl 1): S51-S54.
6 Brown MJ, Palmer CR, Castaigne A, de Leeuw PW, Mancia G, Rosenthal T, Ruilope LM. Morbidity and mortality in patients randomised to double-blind treatment with a longacting calcium-channel blocker or diuretic in the International Nifedipine GITS study: Intervention as a Goal in Hypertension Treatment (INSIGHT). Lancet 2000; 356: 366-372.

7 Hayashi K, Wakino S, Sugano N, Ozawa Y, Homma K, Saruta T. Ca2+ channel subtypes and pharmacology in the kidney. Circ Res 2007; 100: 342-353.

8 ter Wee PM, De Micheli AG, Epstein M. Effects of calcium antagonists on renal hemodynamics and progression of nondiabetic chronic renal disease. Arch Intern Med 1994; 154: 1185-1202.

9 Yamamoto E, Lai ZF, Yamashita T, Tanaka T, Kataoka K, Tokutomi Y, Ito T, Ogawa H, Kim-Mitsuyama S. Enhancement of cardiac oxidative stress by tachycardia and its critical role in cardiac hypertrophy and fibrosis. J Hypertens 2006; 24: 2057-2069.

10 Konno S, Hirooka Y, Araki S, Koga Y, Kishi T, Sunagawa K. Azelnidipine decreases sympathetic nerve activity via antioxidant effect in the rostral ventrolateral medulla of stroke-prone spontaneously hypertensive rats. J Cardiovasc Pharmacol 2008; 52: 555-560.

11 Fujimoto S, Satoh M, Nagasu H, Horike H, Sasaki T, Kashihara N. Azelnidipine exerts renoprotective effects by improvement of renal microcirculation in angiotensin $\mathrm{II}$ infusion rats. Nephrol Dial Transplant 2009; 24: 3651-3658.

12 Nakamura T, Sugaya T, Kawagoe Y, Suzuki T, Ueda Y, Koide H, Inoue T, Node K. Azelnidipine reduces urinary protein excretion and urinary liver-type fatty acid binding protein in patients with hypertensive chronic kidney disease. Am J Med Sci 2007; 333: 321-326.

13 Tuncel M, Augustyniak R, Zhang W, Toto RD, Victor RG. Sympathetic nervous system function in renal hypertension. Curr Hypertens Rep 2002; 4: 229-236.

14 Denton KM, Shweta A, Anderson WP. Preglomerular and postglomerular resistance responses to different levels of sympathetic activation by hypoxia. J Am Soc Nephrol 2002; 13: 27-34.

15 Shokoji T, Fujisawa Y, Kiyomoto H, Rahman M, Sun GP, Fan YY, Kimura S, Kohno M, Abe Y, Nishiyama A. Effects of a new calcium channel blocker, azelnidipine, on systemic hemodynamics and renal sympathetic nerve activity in spontaneously hypertensive rats. Hypertens Res 2005; 28: 1017-1023.

16 Eguchi K, Tomizawa H, Ishikawa J, Hoshide S, Fukuda T, Numao T, Shimada K, Kario $\mathrm{K}$. Effects of new calcium channel blocker, azelnidipine, and amlodipine on baroreflex sensitivity and ambulatory blood pressure. J Cardiovasc Pharmacol 2007; 49: 394-400.

17 Pagani M, Lombardi F, Guzzetti S, Rimoldi O, Furlan R, Pizzinelli P, Sandrone G, Malfatto G, Dell'Orto S, Piccaluga E, Turiel M, Baselli G, Cerutti S, Malliani A. Power spectral analysis of heart rate and arterial pressure variabilities as a marker of sympatho-vagal interaction in man and conscious dog. Circ Res 1986; 59: 178-193.

18 Malliani A, Pagani M, Lombardi F, Cerutti S. Cardiovascular neural regulation explored in the frequency domain. Circulation 1991; 84: 482-492.

19 Heart rate variability. Standards of measurement, physiological interpretation, and clinical use. Task Force of the European Society of Cardiology and the North American Society of Pacing and Electrophysiology. Eur Heart J 1996; 17: 354-381.

20 Nagasu H, Satoh M, Kuwabara A, Yorimitsu D, Sakuta T, Tomita N, Kashihara N. Renal denervation reduces glomerular injury by suppressing NAD(P)H oxidase activity in Dahl salt-sensitive rats. Nephrol Dial Transplant 2010; 25: 2889-2898.

21 Namikoshi T, Tomita N, Satoh M, Haruna Y, Kobayashi S, Komai N, Sasaki T, Kashihara N. Pioglitazone enhances the antihypertensive and renoprotective effects of candesartan in Zucker obese rats fed a high-protein diet. Hypertens Res 2008; 31: 745-755.

22 Reddi AS, Bollineni JS. Selenium-deficient diet induces renal oxidative stress and injury via TGF-beta1 in normal and diabetic rats. Kidney Int 2001; 59: 1342-1353.

23 Fujimoto S, Satoh M, Horike H, Hatta H, Haruna Y, Kobayashi S, Namikoshi T, Arakawa $\mathrm{S}$, Tomita N, Kashihara N. Olmesartan ameliorates progressive glomerular injury in subtotal nephrectomized rats through suppression of superoxide production. Hypertens Res 2008; 31: 305-313.

24 Honda M, Hayashi K, Matsuda H, Kubota E, Tokuyama H, Okubo K, Takamatsu I, Ozawa Y, Saruta T. Divergent renal vasodilator action of $L$ - and T-type calcium antagonists in vivo. J Hypertens 2001; 19: 2031-2037.

25 Ishimitsu T, Kameda T, Akashiba A, Takahashi T, Ohta S, Yoshii M, Minami J, Ono H, Numabe A, Matsuoka $\mathrm{H}$. Efonidipine reduces proteinuria and plasma aldosterone in patients with chronic glomerulonephritis. Hypertens Res 2007; 30: 621-626.

26 Takenaka T, Forster H, De Micheli A, Epstein M. Impaired myogenic responsiveness of renal microvessels in Dahl salt-sensitive rats. Circ Res 1992; 71: 471-480.

27 Huang BS, Wang H, Leenen FH. Enhanced sympathoexcitatory and pressor responses to central $\mathrm{Na}+$ in Dahl salt-sensitive vs. -resistant rats. Am J Physiol Heart Circ Physiol 2001; 281: H1881-H1889.

28 Frohlich ED, McLoughlin MJ, Losem CJ, Ketelhut R, Messerli FH. Hemodynamic comparison of two nifedipine formulations in patients with essential hypertension. Am J Cardiol 1991; 68: 1346-1350.

29 Lukic-Panin V, Kamiya T, Zhang H, Hayashi T, Tsuchiya A, Sehara Y, Deguchi K, Yamashita T, Abe K. Prevention of neuronal damage by calcium channel blockers with antioxidative effects after transient focal ischemia in rats. Brain Res 2007; 1176: 143-150.

30 Yamagishi S, Inagaki Y, Nakamura K, Imaizumi T. Azelnidipine, a newly developed longacting calcium antagonist, inhibits tumor necrosis factor-alpha-induced interleukin-8 expression in endothelial cells through its anti-oxidative properties. J Cardiovasc Pharmacol 2004; 43: 724-730. 
31 Campese VM, Shaohua Y, Huiquin Z. Oxidative stress mediates angiotensin II-dependent stimulation of sympathetic nerve activity. Hypertension 2005; 46: 533-539.

32 Fujita M, Ando K, Nagae A, Fujita T. Sympathoexcitation by oxidative stress in the brain mediates arterial pressure elevation in salt-sensitive hypertension. Hypertension 2007; 50: 360-367.

33 Hirooka Y, Kimura Y, Nozoe M, Sagara Y, Ito K, Sunagawa K. Amlodipine-induced reduction of oxidative stress in the brain is associated with sympatho-inhibitory effects in stroke-prone spontaneously hypertensive rats. Hypertens Res 2006; 29: 49-56.

34 Lindqvist $M$, Kahan T, Melcher A, Ekholm M, Hjemdahl P. Long-term calcium antagonist treatment of human hypertension with mibefradil or amlodipine increases sympathetic nerve activity. J Hypertens 2007; 25: 169-175.

35 Bakris GL, Hart P, Ritz E. Beta blockers in the management of chronic kidney disease. Kidney Int 2006; 70: 1905-1913.

36 Dickerson JE, Hingorani AD, Ashby MJ, Palmer CR, Brown MJ. Optimisation of antihypertensive treatment by crossover rotation of four major classes. Lancet 1999; 353: 2008-2013.
37 Turnbull F, Neal B, Ninomiya T, Algert C, Arima H, Barzi F, Bulpitt C, Chalmers J, Fagard R, Gleason A, Heritier S, Li N, Perkovic V, Woodward M, MacMahon S. Effects of different regimens to lower blood pressure on major cardiovascular events in older and younger adults: meta-analysis of randomised trials. BMJ 2008; 336: 1121-1123.

38 Jamerson K, Weber MA, Bakris GL, Dahlof B, Pitt B, Shi V, Hester A, Gupte J, Gatlin M, Velazquez EJ. Benazepril plus amlodipine or hydrochlorothiazide for hypertension in high-risk patients. N Engl J Med 2008; 359: 2417-2428.

39 Kishi T, Hirooka Y, Konno S, Sunagawa K. Cilnidipine inhibits the sympathetic nerve activity and improves baroreflex sensitivity in patients with hypertension. Clin Exp Hypertens 2009; 31: 241-249.

40 Harada K, Nomura M, Nishikado A, Uehara K, Nakaya Y, Ito S. Clinical efficacy of efonidipine hydrochloride, a T-type calcium channel inhibitor, on sympathetic activities. Circ J 2003; 67: 139-145.

41 Neumann J, Ligtenberg G, Klein II, Koomans HA, Blankestijn PJ. Sympathetic hyperactivity in chronic kidney disease: pathogenesis, clinical relevance, and treatment. Kidney Int 2004; 65: 1568-1576. 